Hardy Ramanujan Journal

Vol. 28(2005) 30-34

\title{
A lower bound concerning subset sums which do not cover all the residues modulo $p$ Jean-Marc DESHOUILLERS ${ }^{1}$
}

\author{
À la mémoire de S. Srinivasan
}

\begin{abstract}
Let $c>\sqrt{2}$ and let $p$ be a prime number. J-M. Deshouillers and G. A. Freiman proved that a subset $\mathcal{A}$ of $\mathbb{Z} / p \mathbb{Z}$, with cardinality larger than $c \sqrt{p}$ and such that its subset sums do not cover $\mathbb{Z} / p \mathbb{Z}$ has an isomorphic image which is rather concentrated; more precisely, there exists $s$ prime to $p$ such that

$$
\sum_{a \in \mathcal{A}}\left\|\frac{a s}{p}\right\|<1+O\left(p^{-1 / 4} \ln p\right),
$$

where the constant implied in the "O" symbol depends on $c$ at most. We show here that there exist a constant $K$ depending on $c$ at most, and such sets $\mathcal{A}$, such that for all $s$ prime to $p$ one has

$$
\sum_{a \in \mathcal{A}}\left\|\frac{a s}{p}\right\|>1+K p^{-1 / 2} .
$$

1 Let $p$ be a prime number and $\mathcal{A}$ be a set of distinct non-zero residue classes modulo $p$. We denote by $\mathcal{A}^{*}$ the set of the subset sums of $\mathcal{A}$, that is to say

$$
\mathcal{A}^{*}=\left\{\sum_{b \in \mathcal{B}} b, \mathcal{B} \subset \mathcal{A}\right\}
$$
\end{abstract}

G. A. Freiman and the author proved (cf. [1]) the following result.

\footnotetext{
${ }^{1}$ Supported by Université Victor Segalen Bordeaux 2 (EA 2961), Université Bordeaux1 and CNRS (UMR 5465)
} 
Theorem 1. Let $c>\sqrt{2}$. Let $p$ be a prime number and $\mathcal{A}$ be a subset of $\mathbb{Z} / p \mathbb{Z}$ with cardinality larger than $c \sqrt{p}$, such that its subset sums do not cover $\mathbb{Z} / p \mathbb{Z}$. There exists s prime to $p$ such that

$$
\sum_{a \in \mathcal{A}}\left\|\frac{a s}{p}\right\|<1+O\left(p^{-1 / 4} \ln p\right) .
$$

In this paper we prove that the error term cannot be arbitrary small. More precisely, we prove the following

Theorem 2. Let $\sqrt{2}<c<2$. There exists a positive real number $K$ such that for all prime number $p$ which is sufficiently large, there exists a subset $\mathcal{A}$ of $\mathbb{Z} / p \mathbb{Z}$ with cardinality larger than $c \sqrt{p}$, such that its subset sums do not cover $\mathbb{Z} / p \mathbb{Z}$, and such that for every $s$ prime to $p$, one has

$$
\sum_{a \in \mathcal{A}}\left\|\frac{a s}{p}\right\|>1+K p^{-1 / 2}
$$

2 Notation When $a$ and $b$ are two real numbers, we denote by $\langle a, b\rangle$ the set of the integers $x$ from the interval $[a, b]$. For a real number $u$, we use the traditional notation $e(u)=\exp (2 \pi i u)$ and $\|u\|=\min _{z \in \mathbb{Z}}|u-z|$; when $b \in \mathbb{Z} / p \mathbb{Z}$, the expression $e(b / p)$ (resp. $\|b / p\|)$ denotes the common value of all the $e(\tilde{b} / p)$ 's (resp. $\|\tilde{b} / p\|)$, where $\tilde{b}$ is any integer representing the class $b$ ; we further let $|b|$ denote the minimum of $|\tilde{b}|$ over all the representative $\tilde{b}$ of $b$, or equivalently $|b|=p\|b / p\|$.

The letter $p$ denotes a prime number which is sufficiently large to satisfy all the implicit or explicit inequalities.

3 A lemma Before embarking on the construction of $\mathcal{A}$, we state and prove a preliminary technical lemma.

Lemma 1. Let $u$ and $k$ be natural integers with $2 \leq u \leq 2 k-3$. Then any integer $v$ in the interval $\left[k+2,2 k^{2}-3 k\right]$ can be expressed as a sum of at most $v / k$ pairwise distinct elements from the interval $[k+2,5 k]$.

Proof of Lemma 1 The lemma is trivial when $k+2 \leq v \leq 5 k$ and we may now assume that $v>5 k$. Let us write $v=2 q k+r$ with $1 \leq q \leq 2 k-4$ and $3 k<r \leq 5 k$, and let us consider two cases 
- if $q$ is even, say $q=2 \ell$, we have $\ell \leq k-2$ and we can write $2 q k=$ $\sum_{|h| \leq \ell, h \neq 0}(2 k+h)$,

- if $q$ is odd, say $q=2 \ell+1$, we have $\ell \leq k-2$ and we can write $2 q k=\sum_{|h| \leq \ell}(2 k+h)$.

In each case, we can represent $v$ as a sum of $q+1$ pairwise distinct integers from the interval $[k+2,5 k]$, whence the result.

\section{Construction of $\mathcal{A}$}

4.1 We first construct an auxiliary suitable set of integers, $\mathcal{E}$. We recall that $\sqrt{2}<c<2$ and let

$$
L=\max \left\{12,\left\lfloor\frac{4+c^{2}}{4-c^{2}}+1\right\rfloor\right\} \text { and } k=\left\lfloor\sqrt{\frac{p}{L^{2}-1}}+1\right\rfloor
$$

we thus have

$$
\left(L^{2}-1\right)\left(k^{2}-4 k+4\right) \leq p \leq\left(L^{2}-1\right)\left(k^{2}-2 k+1\right) .
$$

We consider the set $\mathcal{B}=\langle k+1, L k\rangle$; we have

$$
2 \sum_{b \in \mathcal{B}} b=\left(L^{2}-1\right) k^{2}+(L-1) k
$$

from which one deduces

$$
(0.5 L-1) k-0.5 \leq \sum_{b \in \mathcal{B}} b-(k+1)-(p-1) / 2 \leq\left(L^{2}+0.5 L\right) k .
$$

By Lemma 1, when $p$ is sufficiently large, we can find distinct elements in $\langle k+2,5 k\rangle$ the sum of which is $\sum_{b \in \mathcal{B}} b-(k+1)-(p-1) / 2$; let us denote by $\mathcal{C}$ the set of those elements and let $\mathcal{D}=\mathcal{B} \backslash \mathcal{C}$. The set $\mathcal{D}$ is included in $\langle k+1, L k\rangle$, contains $\{k+1\} \cup\langle 5 k+1, L k\rangle$ and satisfies

$$
S:=\sum_{d \in \mathcal{D}} d=(p-1) / 2+(k+1) .
$$

We finally define $\mathcal{E}$ by

$$
\mathcal{E}=\mathcal{D} \cup\{-d / d \in \mathcal{D} \text { and } d>k+1\}
$$

4.2 Let us now turn our attention to the set $\mathcal{E}^{*}$ in $\mathbb{Z}$. Its largest positive element is $S$ (defined as $\sum_{d \in \mathcal{D}} d=(p-1) / 2+(k+1)$ ), the sum of the positive elements of $\mathcal{E}$. We have a priori two ways to get the largest element in $\mathcal{E}^{*}$ besides the one we just mentioned: either we subtract the smallest 
positive element of $\mathcal{E}$ (which is $k+1$ ), or we add its negative element with the minimal absolute value (which is at most $-(k+2)$ ); there are thus no element of $\mathcal{E}^{*}$ between $S-(k+1)$, which is $(p-1) / 2$ and $S$, which is strictly larger than $(p+3) / 2$. On the other hand, by a similar computation, the smallest element in $\mathcal{E}^{*}$ is the sum of the negative elements of $\mathcal{E}$, which is $-(S-(k+1))=-(p-1) / 2$, and the smallest besides it, is larger than or equal to $-(S-(k+1)-(k+2))=-(p-1) / 2+(k+2)$.

4.3 Let $\mathcal{A}$ be the canonical image of $\mathcal{E}$ on $\mathbb{Z} / p \mathbb{Z}$. We show that $\mathcal{A}^{*}$ does not cover $\mathbb{Z} / p \mathbb{Z}$ : let us consider the point $(p+3) / 2$ (or more correctly, its canonical image in $\mathbb{Z} / p \mathbb{Z})$. The only integers in $\mathcal{E}^{*}$ that can cover this point are $(p+3) / 2$, which impossible, or $(p+3) / 2-p=-(p-3) / 2=-(p-1) / 2+1$, which is again impossible. Thus $\mathcal{A}$ is different from $\mathbb{Z} / p \mathbb{Z}$.

5 No dilation of $\mathcal{A}$ leads to a small sum It remains to show that relation (2) is satisfied.

5.1 We first consider the case when $s$ is 1 or -1 . In this case, we have $\sum_{a \in \mathcal{A}}\|s a / p\|=2(S / p)-(k+1) / p=1+k / p>1+\left(\left(1 / \sqrt{\left(L^{2}-1\right)}\right) \cdot p^{-1 / 2}\right)$.

5.2 When $1<|s|<p /(2 L k)$, we have $\|s a / p\|=|s| .\|a / p\|$ and so $\sum_{a \in \mathcal{A}}\|s a / p\|>|s| .(1+k / p)>2$.

5.3 Let us now consider the case when $p /(2 L k) \leq|s| \leq p /((L-6) k)$. The interval $\langle 5 k+1,6 k\rangle$ is in $\mathcal{D}$ and for any integer $d$ in this interval we have $2 / L<|s| d / p<p / 2$; this implies $\sum_{a \in \mathcal{A}}\|s a / p\|>2 k / L$, which is larger than 2 when $p$ is large enough.

5.4 We finally consider the case when $p /((L-6) k) \leq|s|<p / 2$. For any real number $x$ we have $2 \pi|| x \| \geq 2|\sin (\pi x)| \geq 2 \sin ^{2}(\pi x)=1-\cos (2 \pi x)=$ $1-\Re(e(x))$. Since the interval $\langle 5 k+1, L k\rangle$ is included in $\mathcal{D}$, we have

$$
\begin{aligned}
\sum_{a \in \mathcal{A}}\|s a / p\| & \geq \sum_{h=5 k+1}^{L k}\|s h / p\| \geq \frac{1}{2 \pi} \sum_{h=5 k+1}^{L k}(1-\Re(e(s h / p))) \\
& =\frac{1}{2 \pi}\left((L-5) k-\Re\left(\sum_{h=5 k+1}^{L k} e(s h / p)\right)\right) .
\end{aligned}
$$


We further have

$$
\left|\Re\left(\sum_{h=5 k+1}^{L k} e(s h / p)\right)\right| \leq\left|\sum_{h=5 k+1}^{L k} e(s h / p)\right| \leq \frac{2}{2|\sin (\pi s / p)|},
$$

and since $|s|$ is less than $p / 2$, we have

$$
\left|\Re\left(\sum_{h=5 k+1}^{L k} e(s h / p)\right)\right| \leq \frac{p}{2|s|} \leq(L-6) k .
$$

We thus have

$$
\sum_{a \in \mathcal{A}}\|s a / p\| \geq k /(2 \pi) \geq 2
$$

as soon as $p$ is sufficiently large.

This ends the proof of Theorem 2 .

6 Concluding remarks In order to get a result of the type $\sum_{a \in \mathcal{A}}\left\|\frac{a s}{p}\right\|<$ $1+\Omega\left(p^{-1 / 2}\right)$, we need, with our construction, to have an upper bound for $\operatorname{Card}(\mathcal{A})$ of the type $c \sqrt{p}$ with $c<2$, and we believe that when $c$ tends to 2, such a result cannot be valid.

In the other direction, we conjecture that, in Theorem 1, the upper bound for the error term may replaced by $O\left(p^{-1 / 2}\right)$. However, our construction may be adapted to show that such an error term cannot be valid when $\operatorname{Card}(\mathcal{A})=o\left(p^{-1 / 2}\right)$.

\section{References}

[1] Deshouillers, J-M., Freiman, G. A., When subset sums do not cover all the residues modulo $\mathrm{p}$ 